\title{
Transcriptome-Wide Identification and Expression Profiling Analysis of Chrysanthemum Trihelix Transcription Factors
}

\author{
Aiping Song, Dan Wu, Qingqing Fan, Chang Tian, Sumei Chen, Zhiyong Guan, Jingjing Xin, \\ Kunkun Zhao and Fadi Chen * \\ College of Horticulture, Nanjing Agricultural University, Nanjing 210095, China; \\ aiping_song@aliyun.com (A.S.); 2013104120@njau.edu.cn (D.W.); 2013104100@njau.edu.cn (Q.F.); \\ 2015204031@njau.edu.cn (C.T.); chensm@njau.edu.cn (S.C.); guanzhy@njau.edu.cn (Z.G.); \\ 2014104098@njau.edu.cn (J.X.); 2014104099@njau.edu.cn (K.Z.) \\ * Correspondence: chenfd@njau.edu.cn; Tel.: +86-25-8439-5231; Fax: +86-25-8439-5266 \\ Academic Editor: Lam-Son Phan Tran \\ Received: 19 December 2015; Accepted: 28 January 2016; Published: 2 February 2016
}

\begin{abstract}
Trihelix transcription factors are thought to feature a typical DNA-binding trihelix (helix-loop-helix-loop-helix) domain that binds specifically to the GT motif, a light-responsive DNA element. Members of the trihelix family are known to function in a number of processes in plants. Here, we characterize 20 trihelix family genes in the important ornamental plant chrysanthemum (Chrysanthemum morifolium). Based on transcriptomic data, 20 distinct sequences distributed across four of five groups revealed by a phylogenetic tree were isolated and amplified. The phylogenetic analysis also identified four pairs of orthologous proteins shared by Arabidopsis and chrysanthemum and five pairs of paralogous proteins in chrysanthemum. Conserved motifs in the trihelix proteins shared by Arabidopsis and chrysanthemum were analyzed using MEME, and further bioinformatic analysis revealed that $16 \mathrm{CmTHs}$ can be targeted by 20 miRNA families and that miR414 can target $9 \mathrm{CmTHs}$. qPCR results displayed that most chrysanthemum trihelix genes were highly expressed in inflorescences, while $20 \mathrm{CmTH}$ genes were in response to phytohormone treatments and abiotic stresses. This work improves our understanding of the various functions of trihelix gene family members in response to hormonal stimuli and stress.
\end{abstract}

Keywords: Chrysanthemum morifolium; phylogenetic analysis; stress response; transcription pattern; trihelix

\section{Introduction}

Plant growth and productivity are under constant threat from environmental changes in the form of biotic and abiotic stresses. With a very important role in signal transduction in plants in response to stress [1], transcription factors (TFs) bind to specific gene promoter regions and cis-acting elements to activate or inhibit transcription [2].

There are over 60 TF families in plants [3], and their functions are gradually being defined. A growing number of TFs belonging to such families as MYB [4] and WRKY [5] have been linked to responses to environmental stress in chrysanthemum. In contrast, studies on trihelix TFs are limited to date, though this family has recently attracted attention. This family (also known as GT factors) was named according to its conserved DNA-binding domain, which contains three tandem helices (helix-loop-helix-loop-helix) that bind specifically to the GT motif, a light-responsive DNA element. The DNA-binding domain of GT factors are rich in basic and acidic amino acids, as well as proline and glutamine residues, and GT elements are highly degenerate cis-elements with A/T-rich 
core sequences [6]. The amino acid sequences of these functional domains exhibit a high degree of conservation, also typically with similarity at either the N-terminus or C-terminus.

The trihelix family has been divided into GT-1, GT-2, GT $\gamma$, SH4, and SIP1 5 subfamilies [3], with the name of each clade based on the first member identified. GT-1 clade proteins (GT-1 and GT-3) with one trihelix DNA-binding domain specially bind to Box II (5'-GTGTGGTTAATATG-3') and the 5'-GTTAC-3' motif, respectively [7]. GT-2 types have two DNA TF domains: a C-terminal domain that recognizes the GT-2 box (5'-GCGGTAATTAA- $\left.3^{\prime}\right)$ and an N-terminal domain that recognizes the GT-3 box (5'-GAGGTAAATCCGCGA-3') [8]. The C-terminus of trihelix family members show large differences in structure, often forming a long $\alpha$-helix domain that is predicted to form a coiled-coil structure [9]. Homology analysis reveals higher similarity between the GT-1 and GT-2 clade compared to the others.

Although knowledge of this family was confined to regulation of light-responsive genes [10], recent studies have indicated that the trihelix family also has important functions in different processes of growth and development involving flowers, stomata, trichomes, embryos, and seeds. Trihelix family genes not only participate in disease resistance and salt tolerance, but also in response to cold and drought [11]. In soybean, 63 GT genes were found to respond to stress, with at least 11 involved in responses to biotic or abiotic stress. PETAL LOSS (PTL), belonging to the GT-2 clade, was the first trihelix gene identified as being associated with floral organ morphogenesis [12]. GmGT2B acts both in plant stress tolerance but also reduces sensitivity to abscisic acid (ABA) [13]. Belonging to the GT-2 clade, the GT-2-LIKE1 (GTL1) gene has been identified as a trihelix TF related to trichomes, with increased levels of polyploidy and reduced stomatal numbers occurring with mutations [14]. PtaGTL1 of poplar demonstrates high homology to Arabidopsis AtGTL1 and is also involved in the development of stomata and trichomes [15]. Genetic analyses revealed that the expression of SHAT1 in abscission zone was positively regulated by the trihelix transcription factor $\mathrm{SH} 4$, which is involved in regulating traits of rice grain separation [16]. A gene of the SIP1 family (At3g10030) was reported to be associated with leaf development, as the corresponding mutant displayed a short stature, leaf distortion, and a light green color [17].

Chrysanthemum (Chrysanthemum morifolium Ramat.) is a popular ornamental species that is globally considered second to rose in its market value [18]. With the rapid development of molecular biology, the molecular genetic improvement of chrysanthemum is increasingly becoming a hot topic. As trihelix TFs interact with cis-elements present in the promoter regions of several stress-related genes, regulating their expression to enhance plant stress tolerance, they may play a critical role in stress signaling transduction pathways. Trihelix TFs genes have been cloned in a variety of plants but mainly model plants, such as 30 members in Arabidopsis thaliana and 31 in rice. Conversely, there are few reports related to chrysanthemum. Here, we report the isolation of 20 chrysanthemum trihelix TFs based on a set of transcriptomic data. We performed a comparative phylogenetic analysis of the chrysanthemum genes with those in Arabidopsis. Some plant hormones, such as ABA, salicylic acid (SA), and methyl jasmonate (MeJA), are involved in the response to various stresses by activating the transcription of several defense-related genes. For example, SA and MeJA coordinately function in biotic stress signaling upon pathogen infection [19], and ABA is extensively involved in the response to various biotic and abiotic stresses, including pathogen infection, cold, and drought stress [20]. In order to speculate function of 20 chrysanthemum trihelix TFs, we further analyzed the effect of various stress and phytohormone treatments on transcription levels.

\section{Results}

\subsection{Identification and Phylogenetic Analysis of Putative Trihelix Factors in Chrysanthemum}

Twenty chrysanthemum trihelix gene sequences were isolated and designated $\mathrm{CmTH1}$ through CmTH20 (GenBank: KT253111-KT253130). The full-length cDNAs varied in length from 724 to 2421 bp, with predicted protein products from 211 (CmTH18) to $684(\mathrm{CmTH16})$ amino acids. Details regarding 
the CmTH sequences are given in Table 1. Twelve CmTH proteins are predicted to be nuclearly localized, excepting CmTH4, 6, 7, 9, 10,11, 12, and 18, in which a conserved bipartite nuclear localization signal (NLS) was not found. The predicted localizations are provided in Table 1.

Table 1. Summary of $\mathrm{CmTH}$ sequences and the identity of likely A. thaliana homologs AdMyb/SANT = alcohol dehydrogenase transcription factor Myb/SANT-like family protein HLP = homeodomain-like superfamily protein SSDB-TF = sequence-specific DNA binding transcription factors DHLP = duplicated homeodomain-like superfamily protein. $\mathrm{pI}$, isoelectric point; $M \mathrm{w}$, molecular weight.

\begin{tabular}{|c|c|c|c|c|c|c|c|}
\hline Gene & $\begin{array}{c}\text { GenBank } \\
\text { Accession No. }\end{array}$ & $\begin{array}{l}\text { Amino Acids } \\
\text { Length (aa) }\end{array}$ & $\begin{array}{c}\text { AtTH } \\
\text { Orthologs }\end{array}$ & Locus Name & pI & $M_{\mathrm{W}}$ & $\begin{array}{l}\text { Subcellular } \\
\text { Loclization }\end{array}$ \\
\hline $\mathrm{CmTH1}$ & KT253111 & 340 & $A d M y b / S A N T$ & AT2G44730.1 & 9.51 & $38,618.98$ & nucleus \\
\hline $\mathrm{CmTH} 2$ & KT253112 & 314 & $A d M y b / S A N T$ & AT2G44730.1 & 9.09 & $35,364.01$ & nucleus \\
\hline $\mathrm{CmTH3}$ & KT253113 & 338 & $A d M y b / S A N T$ & AT2G44730.1 & 6.13 & $38,292.92$ & nucleus \\
\hline $\mathrm{CmTH4}$ & KT253114 & 224 & $A d M y b / S A N T$ & AT2G44730.1 & 9.56 & $24,572.87$ & $\begin{array}{l}\text { chloroplast } \\
\text { stroma }\end{array}$ \\
\hline CmTH5 & KT253115 & 295 & $A d M y b / S A N T$ & AT2G44730.1 & 8.52 & $33,510.27$ & nucleus \\
\hline $\mathrm{CmTH6}$ & KT253116 & 422 & ASIL2 & AT3G14180.1 & 9.27 & $47,237.56$ & $\begin{array}{c}\text { chloroplast } \\
\text { thylakoid space }\end{array}$ \\
\hline $\mathrm{CmTH7}$ & KT253117 & 399 & HLP & АT3G25990.1 & 6.85 & $45,568.92$ & microbody \\
\hline $\mathrm{CmTH} 8$ & KT253118 & 249 & $S S D B-T F$ & АT3G54390.1 & 9.30 & $27,119.85$ & nucleus \\
\hline СmTH9 & KT253119 & 446 & $S S D B-T F$ & AT1G21200.1 & 6.35 & $51,562.20$ & cytoplasm \\
\hline CmTH10 & KT253120 & 384 & SSDB-TF & AT1G21200.1 & 6.26 & $43,610.86$ & cytoplasm \\
\hline СmTH11 & KT253121 & 357 & $A d M y b / S A N T$ & АT3G24490.1 & 4.53 & $41,753.36$ & $\begin{array}{l}\text { mitochondrial } \\
\text { matrix space }\end{array}$ \\
\hline CmTH12 & KT253122 & 216 & $S S D B-T F$ & AT5G05550.2 & 9.18 & $24,860.42$ & cytoplasm \\
\hline CmTH13 & KT253123 & 373 & $A d M y b / S A N T$ & АT3G24490.1 & 5.04 & $42,980.38$ & nucleus \\
\hline CmTH14 & KT253124 & 372 & $A d M y b / S A N T$ & АT3G24490.1 & 5.00 & $42,911.32$ & nucleus \\
\hline CmTH15 & KT253125 & 382 & $A d M y b / S A N T$ & АT3G24490.1 & 4.89 & $43,488.77$ & nucleus \\
\hline CmTH16 & KT253126 & 684 & DHLP & AT1G76880.1 & 6.68 & $76,768.06$ & nucleus \\
\hline CmTH17 & KT253127 & 597 & DHLP & AT1G76880.1 & 5.31 & $66,479.62$ & nucleus \\
\hline СmTH18 & KT253128 & 211 & $S S D B-T F$ & AT5G05550.2 & 5.1 & $23,338.93$ & cytoplasm \\
\hline CmTH19 & KT253129 & 341 & $H L P$ & AT5G47660.1 & 9.62 & $39,351.70$ & nucleus \\
\hline $\mathrm{CmTH} 20$ & KT253130 & 526 & DHLP & AT5G28300.1 & 5.75 & $61,139.47$ & nucleus \\
\hline
\end{tabular}

To evaluate evolutionary relationships between Arabidopsis and chrysanthemum TH proteins, the deduced amino acid sequences of the TH genes identified were completely aligned. A combined phylogenetic tree (Figure 1) was then constructed using the neighbor-joining method and bootstrap analysis (1000 reiterations), showing diversification among the plant GT family (Figure 1). The twenty $\mathrm{CmTH}$ genes were found to be distributed across four of five groups, with the exception of the SH4 clade. Furthermore, four pairs of orthologous proteins were identified in Arabidopsis and chrysanthemum, AT3G10030 with CmTH18, AT3G24490 with CmTH11, AT5G47660 with CmTH19, AT5G28300 (AtGT2L) with CmTH20, and five pairs of paralogous trihelix family proteins were identified in chrysanthemum: CmTH1 with $\mathrm{CmTH2}$, CmTH4 with CmTH5, CmTH9 with CmTH10, CmTH13 with CmTH14, and CmTH16 with CmTH17.

\subsection{Conserved Sequences in TH Proteins}

The MEME software was used to predict the motif composition of the trihelix factors, and 10 putative motifs with E values less than $1.8 \times 10^{-45}$ were identified (Figure 2 and Figure S1). The trihelix TFs of chrysanthemum can be clearly classified into five subgroups based on the 
composition of motifs (Figure 2). For example, Motif 2 is mainly shared among the SIP1 clade; Motif 3 is only present in the SIP1 clade, whereas Motif 5 is only absent from this clade. Motif 4 is not found in the SH4 or GT $\gamma$ clade, and Motif 10 is mainly shared among GT $\gamma$ clade members. The GT- 1 and GT-2 subfamilies possess Motif 6, whereas the GT-2 and GT $\gamma$ subfamilies contain Motif 7 (Figure 2). Details on these motif features are shown in Figure S1.

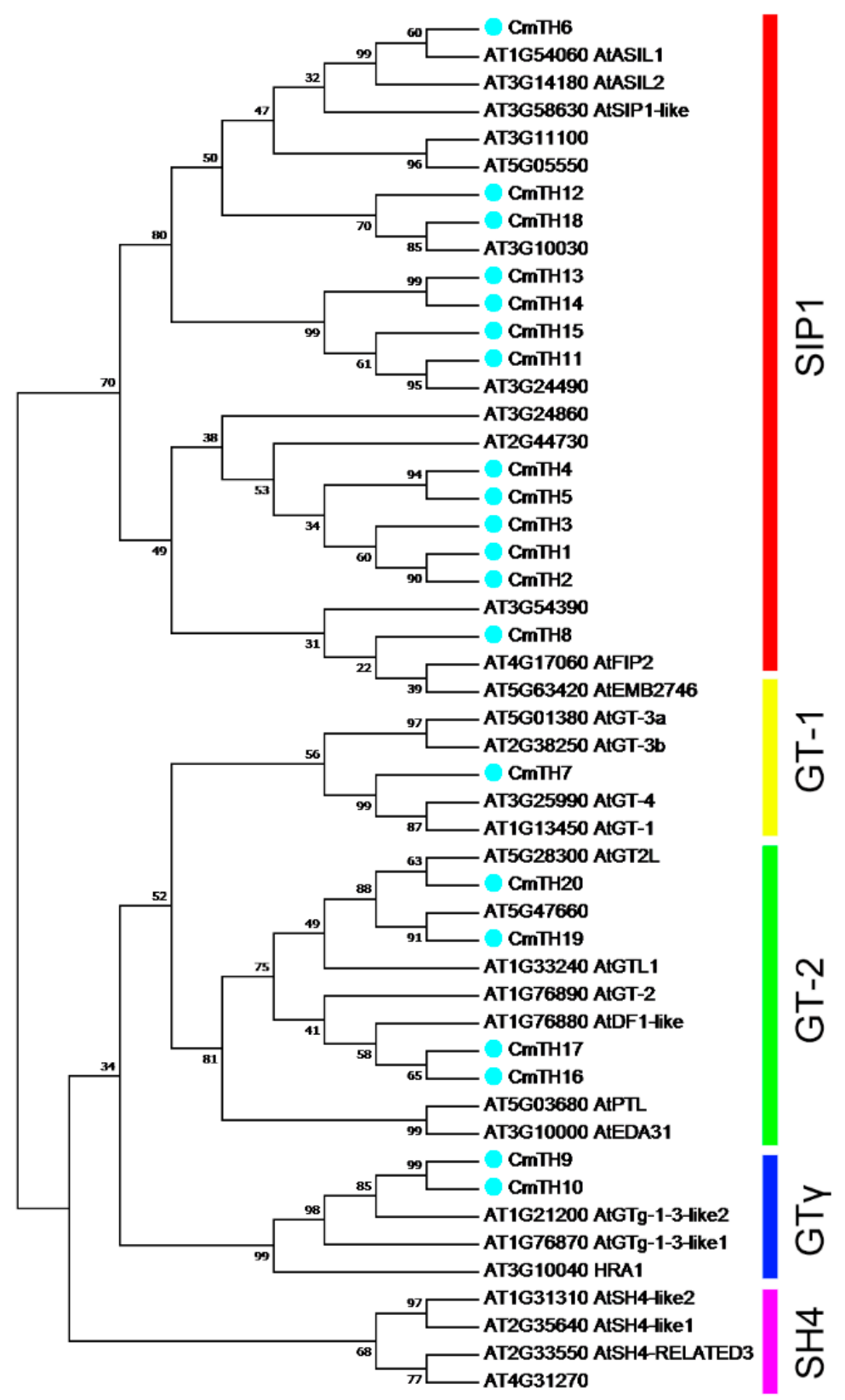

Figure 1. Phylogenetic tree and distribution of conserved motifs among Arabidopsis and chrysanthemum TH proteins. The tree was constructed based on a complete alignment of 30 Arabidopsis and 20 chrysanthemum trihelix proteins using the neighbor-joining method.

The conserved sequences of these motifs were searched against the InterPro database. Motif 1 matched a Myb-type HTH (helix-turn-helix) DNA-binding domain (IPR017930), though no significant matches were retrieved for the other motifs. Most of the $\mathrm{CmTH}$ proteins (except for CmTH4 and CmTH8) contain a Myb-type DNA-binding domain (Motif 1), that is usually located near the N-terminus. 


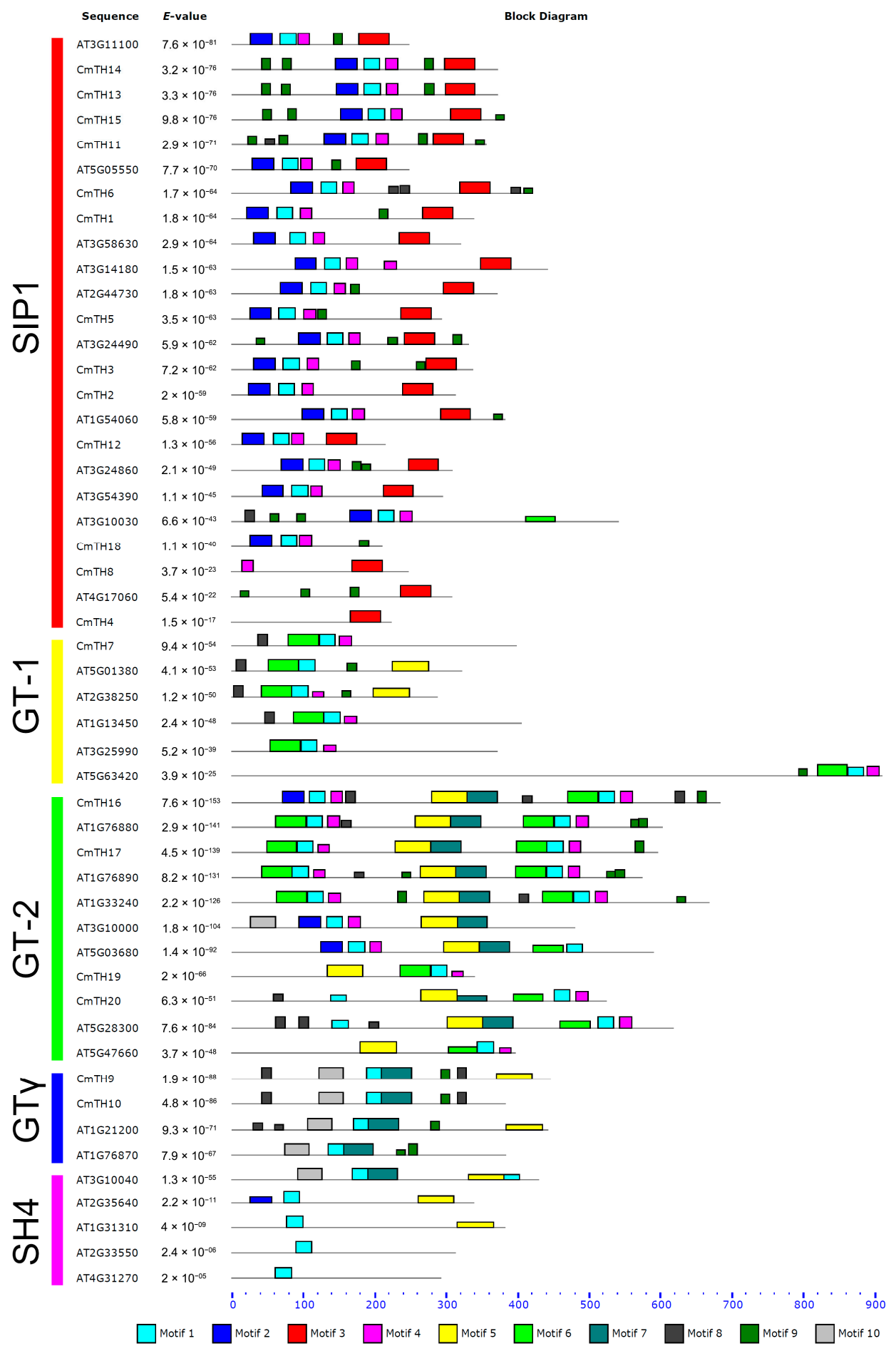

Figure 2. The trihelix protein motifs as derived by MEME analysis. The sequences of motifs of trihelix proteins shown in Figure S1.

\section{3. miRNA Target Site Prediction}

All available plant miRNA data were used to predict candidates targeting $\mathrm{CmTH}$ transcripts. As shown in Table S1, 16 CmTHs are predicted to be targeted by 20 miRNA families, with inhibition of cleavage and/or translation. CmTH14 and CmTH15 contain three target sites and CmTH11, 13 and 17 two target sites; the other $11 \mathrm{CmTHs}$ have only one target site. Additionally, miR414 can target nine of the CmTHs. 


\subsection{Transcription Profiling of CmTH Genes}

Since no trihelix factors in chrysanthemum have been previously documented, we investigated the expression profiles of these genes. The results showed differential expression of the $20 \mathrm{CmTH}$ genes throughout the plant (Figure 3). However, the expression of CmTH11 in ray florets was more than three orders of magnitude higher than that of CmTH15 in roots. Interestingly, the CmTH13 and CmTH14 paralogs exhibited a similar expression pattern, whereas the other four pairs of paralogs showed different patterns.

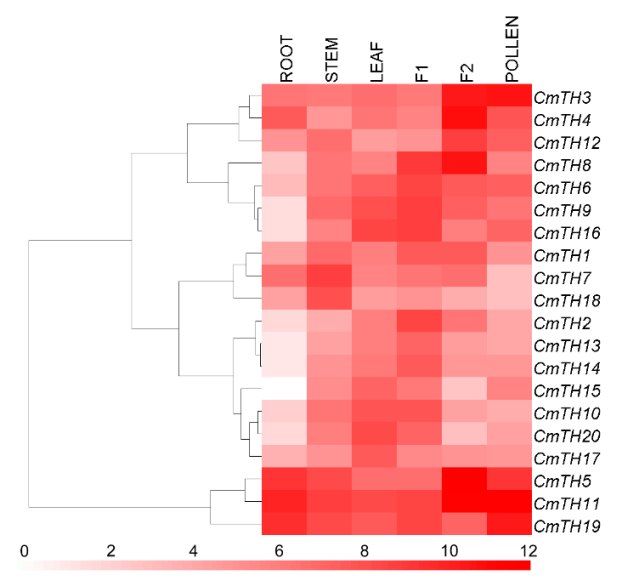

Figure 3. Differential transcription of $\mathrm{CmTH}$ genes. F1: tubular florets, F2: ray florets at the budding stage. White and red indicate lower and higher transcript abundance, respectively. Bar at the bottom represents $\log _{2}$ transformed values.

\subsection{Expression of $\mathrm{CmTH}$ Genes after Treatment with Phytohormones}

Eleven of the twenty $\mathrm{CmTH}$ genes were significantly down-regulated by exogenous ABA, though $\mathrm{CmTH6}, \mathrm{CmTH8}, \mathrm{CmTH12}, \mathrm{CmTH14}$, and CmTH15 were induced at $24 \mathrm{~h}$. In contrast, CmTH19 and $\mathrm{CmTH} 20$ transcripts were increased at $4 / 24$ and $12 / 24 \mathrm{~h}$ after ABA treatment, respectively, whereas the expression of $\mathrm{CmTH1}, \mathrm{CmTH3}, \mathrm{CmTH} 5, \mathrm{CmTH7}, \mathrm{CmTH10}$, CmTH11, and CmTH15 was not affected by ABA (Figure $4 \mathrm{a}$ ). The chrysanthemum $\mathrm{TH}$ family genes displayed three main expression patterns under MeJA treatment: $16 \mathrm{CmTH}$ genes were induced at $24 \mathrm{~h}$, whereas $\mathrm{CmTH} 2$ and CmTH9 were induced at $4 \mathrm{~h}$ but later repressed; conversely, $\mathrm{CmTH19}$ and $\mathrm{CmTH} 20$ were not significantly altered (Figure $4 \mathrm{~b}$ ). As an antagonist of MeJA, SA repressed the expression of the most of the $\mathrm{CmTH}$ genes, except for CmTH12 and CmTH15/19, who were induced at 1 and $4 \mathrm{~h}$, respectively (Figure 4c).
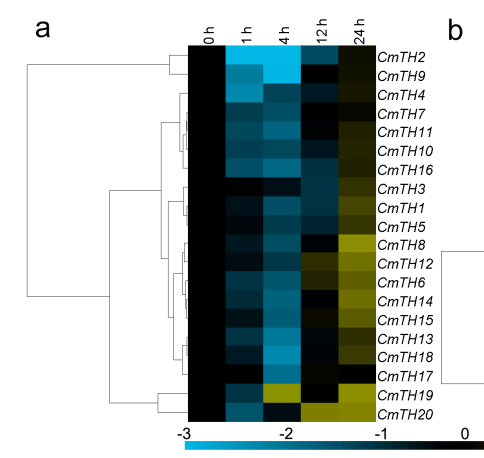

b
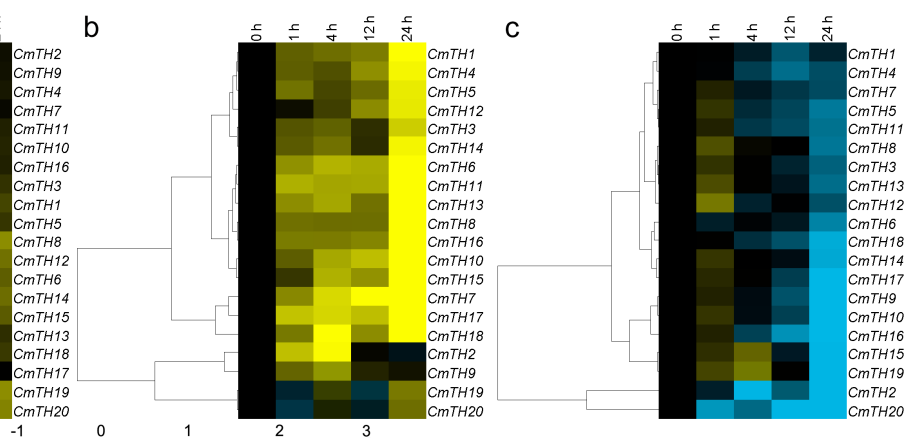

Figure 4. Differential transcription of $\mathrm{CmTH}$ genes in leaves as induced by the exogenous supply of (a) abscisic acid (ABA); (b) methyl jasmonate (MeJA); and (c) salicylic acid (SA) treatments. Blue and yellow indicate lower and higher transcript abundance, respectively, compared to the relevant controls. Bar at the bottom represents $\log _{2}$ transformed values. 


\subsection{Expression Profiling of CmTH Genes under Abiotic Stress}

Three main expression patterns of $\mathrm{CmTH}$ s were observed under salinity stress. Although the expression of $\mathrm{CmTH} 2$ and $\mathrm{CmTH} 16$ was not significantly altered by $\mathrm{NaCl}$ treatment, $\mathrm{CmTH}$ and CmTH14 were suppressed at $24 \mathrm{~h}$; in contrast, the other sixteen CmTHs were up-regulated at 1, 4 and $12 \mathrm{~h}$ (Figure 5a). Six CmTHs (1, 2, 3, 7, 8, and 16) were weakly regulated by drought stress, with a range of variation less than two-fold. CmTH11, CmTH12, CmTH15 and CmTH18 were markedly induced at $1 \mathrm{~h}$ after PEG treatment, whereas eight $\mathrm{CmTHs}(4,6,9,10,13,14,17$, and 20) were only slightly induced. Furthermore, CmTH5 and CmTH19 were up-regulated by high osmotic pressure at $1 \mathrm{~h}$ but were repressed thereafter (Figure 5b). After exposure to low temperature, nine of the $20 \mathrm{CmTH}$ genes (3, $4,5,6,8,16,17,18$, and 19) were strongly suppressed at $4 \mathrm{~h}$. The transcript abundance of CmTH9 was increased at $4 \mathrm{~h}$, and that of $\mathrm{CmTH10}, \mathrm{CmTH11}, \mathrm{CmTH12}, \mathrm{CmTH13}, \mathrm{CmTH14}$, and CmTH15 was also increased at $4 \mathrm{~h}$ but decreased thereafter; the other four CmTHs (1,2, 7, and 20) were weakly regulated by low temperature, with a range of variation less than two-fold (Figure 5c). Eight CmTH genes $(1,2,3$, $9,11,15,16$, and 17) were down-regulated by high temperature, whereas five $(4,5,6,12$, and 20) were up-regulated. The other seven CmTHs were not significantly affected by high temperature (Figure 5d). With the exception of $\mathrm{CmTH12}$, the $\mathrm{CmTH}$ genes were all down-regulated by high temperature, with CmTH4 and CmTH5 transcript expression being decreased more than eight-fold with respect to that at $0 \mathrm{~h}$ (Figure 4g). Five CmTHs (2, 7, 8, 9, and 20) were significantly repressed by mechanical damage, whereas CmTH19 was increased at $1 \mathrm{~h}$ but repressed after $4 \mathrm{~h}$. CmTH15 was up-regulated at 4 and $24 \mathrm{~h}$; CmTH13 and CmTH17 were down-regulated at 24 and $4 \mathrm{~h}$, respectively. The expression of the other $11 \mathrm{CmTHs}$ was not significantly altered by mechanical damage (Figure 5e).

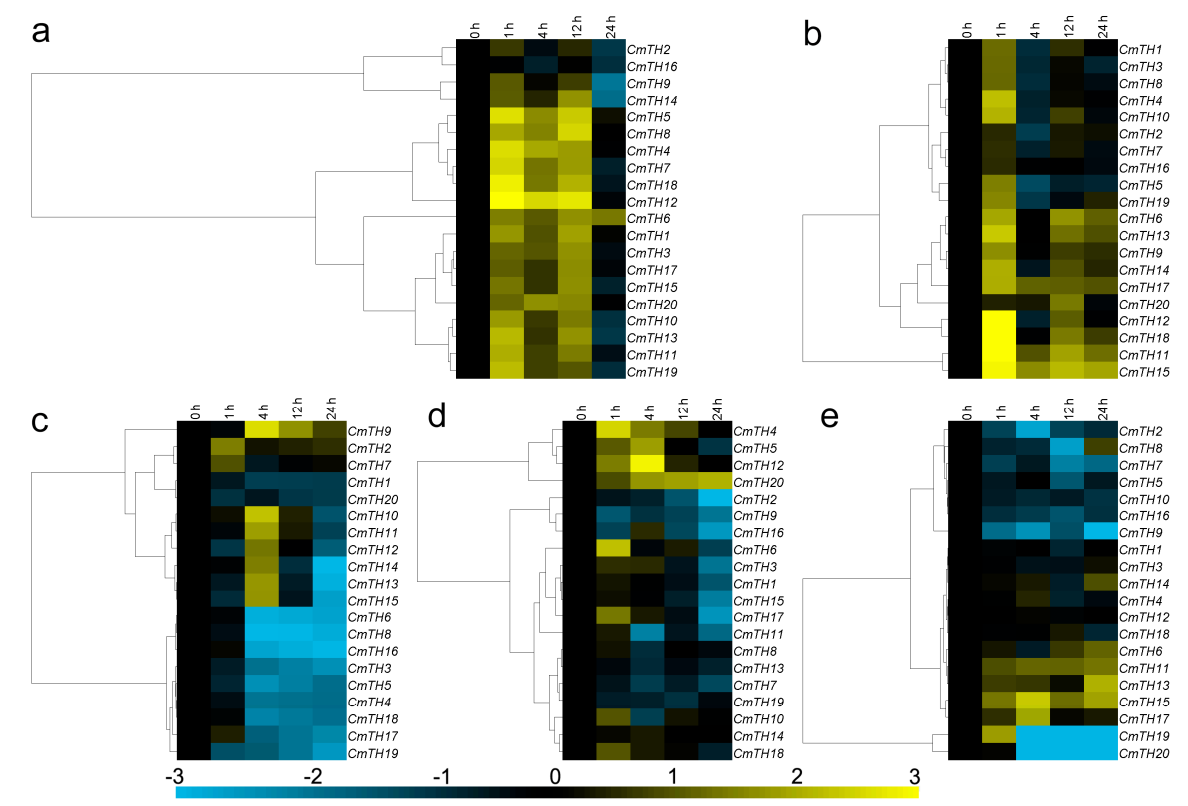

Figure 5. Differential transcription of $\mathrm{CmTH}$ genes in leaves as induced by (a) salinity stress; (b) drought stress; (c) low temperature $\left(4^{\circ} \mathrm{C}\right)$; $(\mathbf{d})$ high temperature $\left(40^{\circ} \mathrm{C}\right)$; and $(\mathbf{e})$ wound treatments. Blue and yellow indicate lower and higher transcript abundance, respectively, compared to the relevant controls. Bar at the bottom represents $\log _{2}$ transformed values.

\section{Discussion}

\subsection{The Trihelix Family in Chrysanthemum}

In general, trihelix TFs are thought to feature a typical DNA-binding trihelix (helix-loop-helixloop-helix) structure [21]. A few trihelix genes from various plants have recently been cloned and 
characterized, showing great functional divergence in processes such as seed scattering during crop domestication, embryo development, morphogenesis control of various flower organs, and resistance to biotic and abiotic stresses [22]. Most of these functions have been studied in Arabidopsis, whereas no members of the family have been isolated from flower crops, e.g., chrysanthemum. By taking advantage of the availability of the chrysanthemum transcriptome, we identified 20 members of this family on the basis of sequence homology using putative trihelix genes as blast queries. The trihelix family has been classified based on the genes in Arabidopsis into five distinct subfamilies: GT-1, GT-2, GT $\gamma$, SIP1, and SH4 [3]. In the present study, we compared Arabidopsis and chrysanthemum sequences and constructed a phylogenetic tree using the neighbor-joining procedure to classify the genes into five major clades (Figure 1). The results are consistent with those of Kaplan-Levy [3]. Our motif analysis also confirmed the classification, with each clade containing specific motifs (Figure 2). Three Arabidopsis genes and two from chrysanthemum (CmTH9 and CmTH10) are found in clade GT $\gamma$, though the function of Arabidopsis genes have not been reported in this clade previously. After searching the TAIR database, 13 out of 30 Arabidopsis trihelix family proteins were located in the nucleus, six proteins were located in the nucleus and other cellular components, nine proteins were located in other cellular components, and two proteins' subcellular localization was unknown. Our result was consistent with it, assessment of the subcellular localization of chrysanthemum trihelix genes revealed strong support (twelve genes) for their functional roles in transcriptional regulation (Table 1). Nonetheless, the transcriptional activity of these family members needs to be investigated further.

\subsection{Diverse Motifs Predicted in TH Factors}

We further analyzed conserved motifs among chrysanthemum trihelix family members using the MEME program and found that the majority of the CmTHs in the same group shared similar motifs, suggesting that these conserved motifs play crucial roles in group-specific functions. However, high divergence in structure was found among the different groups. For example, the GT- 1 and GT-2 subfamilies contain Motif 6, whereas the GT-2 and GT $\gamma$ subfamilies contain Motif 7 (Figure 2), which reflects the complex nature of the function of $\mathrm{TH}$ proteins in chrysanthemum. We also found motifs conserved in certain subfamilies, e.g., Motif 3 in the SIP1 clade and Motif 10 in the GT $\gamma$ clade. The distribution of motifs indicated that the genes containing the same motifs were likely produced through gene expansion within the same group.

Since they all form a helix-turn-helix structure, trihelix TFs are classified into the Myb/SANT-like family (PF13837) in Pfam. In our study, Motif 1 matched the Myb-type HTH (helix-turn-helix) DNA-binding domain (IPR017930). However, the trihelix family is generally thought to feature a typical helix-loop-helix-loop-helix structure with individual helices longer than the myb repeat, which targets different DNA sequences [23].

\section{3. miRNA Target Site Prediction}

To our knowledge, reports on miRNA-TH interactions are rare. Our predictions indicated that $16 \mathrm{CmTHs}$ can be targeted by 20 miRNA families, but only three Arabidopsis GT-2 subfamily genes can be targeted by 5 miRNA families (Table S1). Moreover, we predicted that miR414 can target nine CmTHs (distributed among three subfamilies, GT-2, GT $\gamma$, and SIP1), which suggests that this interaction module is conserved in the chrysanthemum trihelix family. Regardless, these interaction regulation pathways in plants should be verified by further research.

\subsection{Organ-Preferential Expression of CmTH Genes}

Since gene expression patterns can provide important clues for gene function, we employed qRT-PCR to examine the expression of $\mathrm{CmTH}$ genes in the roots, stems and leaves of young plants as well as in the tube and ray florets of inflorescences at the bud stage and in pollen (Figure 3). The expression profiles obtained reveal spatial variations of $\mathrm{CmTH}$ expression in different organs. Furthermore, five pairs of paralogous genes (except CmTH13 and CmTH14) showed distinct 
expression patterns, suggesting that significant functional divergence might have occurred following duplication events.

As shown in Figure 3, most chrysanthemum trihelix genes were highly expressed in inflorescences, which is consistent with previously described functional roles of trihelix genes during flower development [3]. CmTH1, $\mathrm{CmTH7}$, and $\mathrm{CmTH1}$ showed relatively high expression levels in stems, whereas $\mathrm{CmTH} 17$ and $\mathrm{CmTH} 20$ were highly expressed in leaves, suggesting that they might play a role in the development of the stem or leaf, respectively. However, additional research is needed to determine the functions of these $\mathrm{CmTH}$ genes.

\subsection{Transcriptional Responses of Chrysanthemum Trihelix Genes after Phytohormone or against Abiotic Stress Treatment}

As plant trihelix family TFs play very important roles in development and stress response [24], we investigated the responses of $\mathrm{CmTHs}$ to different plant hormones and abiotic stresses. CmTHs were both up-regulated and down-regulated by the treatments (Figures 4 and 5), indicating that $\mathrm{CmTHs}$ may be involved in responses to various plant hormones that elicit a stress response.

In Arabidopsis, GT-1 and related GT-4 are expressed ubiquitously [25], and their homolog in chrysanthemum, $\mathrm{CmTH7}$, is also constitutively expressed in various organs. Arabidopsis GT-2-LIKE1 function was revealed to limit the extent of endoreduplication by modulating the expression of relevant cell cycle genes [26], and the expression of GT-2-LIKE1 is reduced during drought stress, possibly leading to the generation of fewer stomata in newly arising leaves [27]. On the other hand, soybean GmGT-2B is most closely related to GT-2-LIKE1, its expression is induced by ABA, high salt, drought, and cold in 15-day-old seedlings [11]. In chrysanthemum, its ortholog, $\mathrm{CmTH20}$, was found to be induced by $\mathrm{ABA}, \mathrm{NaCl}, \mathrm{PEG}$, and high-temperature treatments, suggesting that it may have functions similar to soybean GmGT-2B, as opposed to Arabidopsis GT-2-LIKE1.

The functions of the GT $\gamma$ clade have only recently been investigated. Expression of OsGT $\gamma-1$, OsGT $\gamma-2$, and $O s G T \gamma-3$ in rice seedlings can be induced by salt, drought, cold, and ABA treatments [28]. There are three GT $\gamma$ group genes in Arabidopsis, though expression of these genes does not appear to exhibit similar stress-induced trends [29]. Our expression data showed induction of GT $\gamma$-clade genes in chrysanthemum ( $\mathrm{CmTH} 9$ and $\mathrm{CmTH10}$ ) by salt, drought, cold, and JA treatments, and further studies on the extent of such stress-related functions will be of interest. The first member of the SIP1 clade, NtSIP1, was identified in tobacco [30], and two SIP1 group genes (ASIL1 and ASIL2) from Arabidopsis have recently been shown to repress the expression of late embryo development genes in seedlings [31]. However, their responses to abiotic and biotic stresses have not been reported to date. Thirteen of twenty chrysanthemum trihelix genes belong to the SIP1 clade, and their transcriptional responses under phytohormone or abiotic stress treatments were analyzed. For instance, most of CmTHs in SIP1 clade (except $\mathrm{CmTH} 2$ and CmTH14) were induced by NaCl stress, furthermore, $\mathrm{CmTH11},-12,-15$ and -18 were markedly induced by PEG treatment. It strongly suggested that SIP1 clade CmTH genes were participated in the abiotic stresses response of chrysanthemum. Our results will promote future research on the biological functions of genes in this clade.

\section{Materials and Methods}

\subsection{Plant Materials and Growth Conditions}

Cuttings of the cut-flower chrysanthemum cultivar "Jinba", maintained at the Chrysanthemum Germplasm Resource Preservation Center (Nanjing Agricultural University, Nanjing, China), were rooted in vermiculite without fertilizer in a greenhouse. After 14 days, the plants were transplanted to a growth substrate and subjected to a range of stress and phytohormone treatments.

\subsection{Database Searches and Sequencing of Full-Length CmTH cDNAs}

All of the putative trihelix proteins were retrieved from C. morifolium transcriptome data, which was derived from petal and leaf tissues [32]. Arabidopsis trihelix protein sequences were downloaded 
from The Arabidopsis Information Resource (TAIR) database and used as query sequences to identify $\mathrm{CmTH}$ proteins. Multiple alignments among the identified $\mathrm{CmTH}$ sequences were also performed to avoid repetition. Furthermore, the full open reading frames of $\mathrm{CmTHs}$ were obtained via RACE PCR. First-strand CDNA was synthesized using the dT adaptor primer dT-AP and then subjected to nested PCR using the primer pair CmTHx-3-F1/F2 and the adaptor primer AP (Table S2). Finally, the complete open reading frames (ORF) of $20 \mathrm{CmTH}$ s were amplified using Phusion ${ }^{\circledR}$ High-Fidelity PCR kit with gene-specific primers (Table S3). The amplicons were purified using AxyPrep DNA Gel Extraction Kit (Axygen, Hangzhou, China) and cloned into pMD19-T (TaKaRa, Tokyo, Japan) for sequencing. The consistent sequence of each $\mathrm{CmTH}$ was used to subsequent analysis.

\subsection{Phylogenetic Tree Construction and Sequence Analysis}

A phylogenetic tree was constructed with MEGA version 6.0 using the neighbor-joining method [33]. ClustalW software was employed for multi-sequence alignments of trihelix TFs between Arabidopsis and C. morifolium [34]. Computation of the theoretical isoelectric point (pI) and molecular weight $(\mathrm{Mw})$ of $\mathrm{CmTH}$ proteins was performed using the Compute $\mathrm{pI} / \mathrm{Mw}$ online tool [35], and PSORT [36] was used to predict subcellular localization. Putative conserved motifs in these collected trihelix proteins were predicted using the MEME program v4.10.2 [37]; the number of motifs was set to 10. All motifs identified by MEME were searched in the InterPro database using Inter-ProScan [38]. Target prediction for miRNA was performed using the psRNATarget online tool [39].

\subsection{Plant Treatments}

Tissue-specific transcription profiles of $20 \mathrm{CmTH}$ genes were explored in roots, stems, and leaves of young plant,s as well as in tube and ray florets of inflorescences at the bud stage and pollen.

A variety of abiotic stresses were imposed, including high salinity $(200 \mathrm{mM} \mathrm{NaCl})$ and drought (20\% w/v polyethylene glycol (PEG6000)) [40]. For NaCl and PEG6000 assays, young plants were transferred to liquid medium containing the stress agent, and the second true leaves were sampled at various time points [41]. Other plants were subjected to a period of exposure at either 4 or $40{ }^{\circ} \mathrm{C}$ in a chamber under a $16 \mathrm{~h}$ photoperiod with $50 \mathrm{\mu mol} \cdot \mathrm{m}^{-2} \cdot \mathrm{s}^{-1}$ of light; the second true leaves were sampled [42]. A wounding treatment involved cutting the second true leaf, and phytohormone treatments involved spraying the leaves with either $50 \mu \mathrm{M}$ ABA, $1 \mathrm{mM}$ MeJA, or $200 \mu \mathrm{M} \mathrm{SA}$ [43]. For all treatments, the second true leaf of each plant was sampled with three biological replicates, prior to stress treatment and then at $1,4,12$, and $24 \mathrm{~h}$.

After sampling, all of the collected material was snap frozen in liquid nitrogen and stored at $-70^{\circ} \mathrm{C}$. Each treatment was replicated three times.

\subsection{Real-Time Quantitative PCR ( $q P C R$ )}

Total RNA was isolated from samples using the RNAiso reagent (TaKaRa) according to the manufacturer's instructions. The RNA was then treated with RNase-free DNase I (TaKaRa) to remove potential genomic DNA contamination. First-strand cDNA was synthesized from $1 \mu \mathrm{g}$ of total RNA using SuperScript III reverse transcriptase (Invitrogen, Carlsbad, CA, USA) according to the manufacturer's instructions. qPCR was performed using a Mastercycler EP Realplex instrument (Eppendorf, Hamburg, Germany). Each $20 \mu \mathrm{L}$ amplification reaction contained $10 \mu \mathrm{L}$ SYBR ${ }^{\circledR}$ Premix Ex Taq $^{\text {TM }}$ II (TakaRa), $0.4 \mu \mathrm{L}$ each primer $(10 \mu \mathrm{M}), 4.2 \mu \mathrm{L} \mathrm{H}_{2} \mathrm{O}$ and $5 \mu \mathrm{L}$ cDNA template. The PCR cycling regime consisted of an initial denaturation $\left(95^{\circ} \mathrm{C} / 2 \mathrm{~min}\right)$ followed by 40 cycles of $95^{\circ} \mathrm{C}$ for $10 \mathrm{~s}, 55^{\circ} \mathrm{C}$ for $15 \mathrm{~s}$, and $72{ }^{\circ} \mathrm{C}$ for $20 \mathrm{~s}$. A melting curve analysis was performed following each assay to confirm the specificity of the amplicons. Gene-specific primers (provided in Table S4) were designed using Primer3 Release 2.3.4 [44], and the EF1 $\alpha$ gene was employed as a reference sequence [43]. Relative transcript abundance was calculated by the $2^{-\Delta \Delta C t}$ method [45]. For each biological replicates, three technical repeats were performed. 


\subsection{Data Analysis}

The relative expression levels of each $\mathrm{CmTH}$ gene were $\log _{2}$ transformed. The profiles were compared using Cluster v3.0 software [46] and visualized using Treeview [47]. The expression data were analyzed by Student's $t$-test using the SPSS v17.0 software (SPSS Inc., Chicago, IL, USA).

\section{Conclusions}

This study is the first transcriptome-wide analysis of the trihelix TF family in chrysanthemum. The phylogenetic analysis revealed twenty $\mathrm{CmTH}$ genes were distributed across four of five groups (except SH4), while transcriptional analysis displayed the expression of $20 \mathrm{CmTHs}$ was in response to a range of phytohormones and abiotic stress treatments. Additionally, bioinformatics analysis predicted that $16 \mathrm{CmTHs}$ can be targeted by 20 miRNA families and miR414 can target nine CmTHs. Our findings lay a foundation for future research on the biological functions of the members of this family, including localized growth suppression and responses to abiotic stresses. Future studies on $\mathrm{CmTH}$ genes will shed light on the fundamental functions of these genes and promote their application in chrysanthemum breeding.

Supplementary Materials: Supplementary materials can be found at http://www.mdpi.com/1422-0067/17/ 2/198/s1.

Acknowledgments: This study was funded by the National Science Fund for Distinguished Young Scholars (31425022), the Fundamental Research Funds for the Central Universities (KJQN201658, KYTZ201401), National Natural Science Foundation of China (31501792), Special Fund for Agro-scientific Research in the Public Interest (201403039), the Natural Science Fund of Jiangsu Province (BK20150657), the China Postdoctoral Science Foundation (2014M561673, 2015T80564), the New agricultural varieties, technology and new model update project of Jiangsu Province (SXGC(2015)322) and the Project Funded by the Priority Academic Program Development of Jiangsu Higher Education.

Author Contributions: Aiping Song, Fadi Chen and Chang Tian conceived and designed the experiments; Qingqing Fan, Jingjing Xin and Kunkun Zhao performed the experiments; Aiping Song and Fadi Chen analyzed the data; Fadi Chen, Sumei Chen and Zhiyong Guan contributed reagents/materials/analysis tools; Aiping Song and Dan Wu wrote the paper.

Conflicts of Interest: The authors declare no conflict of interest.

\section{References}

1. Riechmann, J.; Heard, J.; Martin, G.; Reuber, L.; Jiang, C.-Z.; Keddie, J.; Adam, L.; Pineda, O.; Ratcliffe, O.; Samaha, R. Arabidopsis transcription factors: Genome-wide comparative analysis among eukaryotes. Science 2000, 290, 2105-2110. [CrossRef] [PubMed]

2. Mizoi, J.; Shinozaki, K.; Yamaguchi-Shinozaki, K. AP2/ERF family transcription factors in plant abiotic stress responses. BBA Gene Regul. Mech. 2012, 1819, 86-96. [CrossRef] [PubMed]

3. Kaplan-Levy, R.N.; Brewer, P.B.; Quon, T.; Smyth, D.R. The trihelix family of transcription factors-Light, stress and development. Trends Plant Sci. 2012, 17, 163-171. [CrossRef] [PubMed]

4. Zhu, L.; Shan, H.; Chen, S.; Jiang, J.; Gu, C.; Zhou, G.; Chen, Y.; Song, A.; Chen, F. The heterologous expression of the chrysanthemum R2R3-MYB transcription factor CmMYB1 alters lignin composition and represses flavonoid synthesis in Arabidopsis thaliana. PLoS ONE 2013, 8, e65680. [CrossRef] [PubMed]

5. Li, P.; Song, A.; Gao, C.; Wang, L.; Wang, Y.; Sun, J.; Jiang, J.; Chen, F.; Chen, S. Chrysanthemum WRKY gene CmWRKY17 negatively regulates salt stress tolerance in transgenic chrysanthemum and Arabidopsis plants. Plant Cell Rep. 2015, 34, 1365-1378. [CrossRef] [PubMed]

6. Wang, R.; Hong, G.; Han, B. Transcript abundance of $r m l 1$, encoding a putative GT1-like factor in rice, is up-regulated by Magnaporthe grisea and down-regulated by light. Gene 2004, 324, 105-115. [CrossRef] [PubMed]

7. Wang, X.H.; Li, Q.T.; Chen, H.W.; Zhang, W.K.; Ma, B.; Chen, S.Y.; Zhang, J.S. Trihelix transcription factor GT-4 mediates salt tolerance via interaction with TEM2 in Arabidopsis. BMC Plant Biol. 2014, 14, 339. [CrossRef] [PubMed]

8. Nagano, Y. Several features of the GT-factor trihelix domain resemble those of the Myb DNA-binding domain. Plant Physiol. 2000, 124, 491-494. [CrossRef] [PubMed] 
9. Kuhn, R.M.; Caspar, T.; Dehesh, K.; Quail, P.H. DNA binding factor GT-2 from Arabidopsis. Plant Mol. Biol. 1993, 23, 337-348. [CrossRef]

10. Zhou, D.-X. Regulatory mechanism of plant gene transcription by GT-elements and GT-factors. Trends Plant Sci. 1999, 4, 210-214. [CrossRef]

11. Xie, Z.-M.; Zou, H.-F.; Lei, G.; Wei, W.; Zhou, Q.-Y.; Niu, C.-F.; Liao, Y.; Tian, A.-G.; Ma, B.; Zhang, W.-K. Soybean trihelix transcription factors GmGT-2A and GmGT-2B improve plant tolerance to abiotic stresses in transgenic Arabidopsis. PLoS ONE 2009, 4, e6898. [CrossRef] [PubMed]

12. Li, X.; Qin, G.; Chen, Z.; Gu, H.; Qu, L.-J. A gain-of-function mutation of transcriptional factor PTL results in curly leaves, dwarfism and male sterility by affecting auxin homeostasis. Plant Mol. Biol. 2008, 66, 315-327. [CrossRef] [PubMed]

13. Suprunova, T.; Krugman, T.; Distelfeld, A.; Fahima, T.; Nevo, E.; Korol, A. Identification of a novel gene (Hsdr4) involved in water-stress tolerance in wild barley. Plant Mol. Biol. 2007, 64, 17-34. [CrossRef] [PubMed]

14. Perisic, O.; Lam, E. A tobacco DNA binding protein that interacts with a light-responsive box II element. Plant Cell 1992, 4, 831-838. [CrossRef] [PubMed]

15. Weng, H.; Yoo, C.Y.; Gosney, M.J.; Hasegawa, P.M.; Mickelbart, M.V. Poplar GTL1 is a Ca ${ }^{2+} /$ calmodulin-binding transcription factor that functions in plant water use efficiency and drought tolerance. PLoS ONE 2012, 7, e32925. [CrossRef] [PubMed]

16. Zhou, Y.; Lu, D.; Li, C.; Luo, J.; Zhu, B.-F.; Zhu, J.; Shangguan, Y.; Wang, Z.; Sang, T.; Zhou, B. Genetic control of seed shattering in rice by the APETALA2 transcription factor SHATTERING ABORTION1. Plant Cell 2012, 24, 1034-1048. [CrossRef] [PubMed]

17. Kuromori, T.; Wada, T.; Kamiya, A.; Yuguchi, M.; Yokouchi, T.; Imura, Y.; Takabe, H.; Sakurai, T.; Akiyama, K.; Hirayama, T. A trial of phenome analysis using $4000 \mathrm{Ds}$-insertional mutants in gene-coding regions of Arabidopsis. Plant J. 2006, 47, 640-651. [CrossRef] [PubMed]

18. Song, A.; You, Y.; Chen, F.; Li, P.; Jiang, J.; Chen, S. A multiplex RT-PCR for rapid and simultaneous detection of viruses and viroids in chrysanthemum. Lett. Appl. Microbiol. 2013, 56, 8-13. [CrossRef]

19. Vos, I.A.; Moritz, L.; Pieterse, C.M.; van Wees, S.C. Impact of hormonal crosstalk on plant resistance and fitness under multi-attacker conditions. Front. Plant Sci. 2015, 6, 639. [CrossRef] [PubMed]

20. Lim, C.W.; Baek, W.; Jung, J.; Kim, J.-H.; Lee, S.C. Function of ABA in stomatal defense against biotic and drought stresses. Int. J. Mol. Sci. 2015, 16, 15251-15270. [CrossRef] [PubMed]

21. Nagano, Y.; Inaba, T.; Furuhashi, H.; Sasaki, Y. Trihelix DNA-binding protein with specificities for two distinctcis-elements both important for light down-regulated and dark-inducible gene expression in higher plants. J. Biol. Chem. 2001, 276, 22238-22243. [CrossRef] [PubMed]

22. Barr, M.S.; Willmann, M.R.; Jenik, P.D. Is there a role for trihelix transcription factors in embryo maturation? Plant Signal. Behav. 2012, 7, 205-209. [CrossRef] [PubMed]

23. Nagata, T.; Niyada, E.; Fujimoto, N.; Nagasaki, Y.; Noto, K.; Miyanoiri, Y.; Murata, J.; Hiratsuka, K.; Katahira, M. Solution structures of the trihelix DNA-binding domains of the wild-type and a phosphomimetic mutant of Arabidopsis GT-1: Mechanism for an increase in DNA-binding affinity through phosphorylation. Proteins 2010, 78, 3033-3047. [CrossRef] [PubMed]

24. Yu, C.; Cai, X.; Ye, Z.; Li, H. Genome-wide identification and expression profiling analysis of trihelix gene family in tomato. Biochem. Biophys. Res. Commun. 2015, 468, 653-659. [CrossRef] [PubMed]

25. Murata, J.; Takase, H.; Hiratsuka, K. Characterization of a novel GT-box binding protein from Arabidopsis. Plant Biotechnol. 2002, 19, 103-112. [CrossRef]

26. Breuer, C.; Kawamura, A.; Ichikawa, T.; Tominaga-Wada, R.; Wada, T.; Kondou, Y.; Muto, S.; Matsui, M.; Sugimoto, K. The trihelix transcription factor GTL1 regulates ploidy-dependent cell growth in the Arabidopsis trichome. Plant Cell 2009, 21, 2307-2322. [CrossRef] [PubMed]

27. Yoo, C.Y.; Pence, H.E.; Jin, J.B.; Miura, K.; Gosney, M.J.; Hasegawa, P.M.; Mickelbart, M.V. The Arabidopsis GTL1 transcription factor regulates water use efficiency and drought tolerance by modulating stomatal density via transrepression of SDD1. Plant Cell 2010, 22, 4128-4141. [CrossRef] [PubMed]

28. Fang, Y.; Xie, K.; Hou, X.; Hu, H.; Xiong, L. Systematic analysis of GT factor family of rice reveals a novel subfamily involved in stress responses. Mol. Genet. Genom. 2010, 283, 157-169. [CrossRef] [PubMed] 
29. Schmid, M.; Davison, T.S.; Henz, S.R.; Pape, U.J.; Demar, M.; Vingron, M.; Schölkopf, B.; Weigel, D.; Lohmann, J.U. A gene expression map of Arabidopsis thaliana development. Nat. Genet. 2005, 37, 501-506. [CrossRef] [PubMed]

30. Kitakura, S.; Fujita, T.; Ueno, Y.; Terakura, S.; Wabiko, H.; Machida, Y. The protein encoded by oncogene $6 b$ from Agrobacterium tumefaciens interacts with a nuclear protein of tobacco. Plant Cell 2002, 14, 451-463. [CrossRef] [PubMed]

31. Gao, M.-J.; Lydiate, D.J.; Li, X.; Lui, H.; Gjetvaj, B.; Hegedus, D.D.; Rozwadowski, K. Repression of seed maturation genes by a trihelix transcriptional repressor in Arabidopsis seedlings. Plant Cell 2009, 21, 54-71. [CrossRef] [PubMed]

32. Zhang, F.; Wang, Z.; Dong, W.; Sun, C.; Wang, H.; Song, A.; He, L.; Fang, W.; Chen, F.; Teng, N. Transcriptomic and proteomic analysis reveals mechanisms of embryo abortion during chrysanthemum cross breeding. Sci. Rep. 2014, 4, 6536. [CrossRef] [PubMed]

33. Tamura, K.; Stecher, G.; Peterson, D.; Filipski, A.; Kumar, S. MEGA6: Molecular evolutionary genetics analysis version 6.0. Mol. Biol. Evol. 2013, 30, 2725-2729. [CrossRef] [PubMed]

34. Larkin, M.A.; Blackshields, G.; Brown, N.P.; Chenna, R.; McGettigan, P.A.; McWilliam, H.; Valentin, F.; Wallace, I.M.; Wilm, A.; Lopez, R.; et al. Clustal W and Clustal X version 2.0. Bioinformatics 2007, 23, 2947-2948. [CrossRef] [PubMed]

35. Gasteiger, E.; Hoogland, C.; Gattiker, A.; Duvaud, S.; Wilkins, M.R.; Appel, R.D.; Bairoch, A. Protein identification and analysis tools on the ExPASy server. In The Proteomics Protocols Handbook; Walker, J.M., Ed.; Humana Press: New York City, NY, USA, 2005; pp. 571-607.

36. Horton, P.; Park, K.-J.; Obayashi, T.; Fujita, N.; Harada, H.; Adams-Collier, C.; Nakai, K. WoLF PSORT: Protein localization predictor. Nucleic Acids Res. 2007, 35, W585-W587. [CrossRef]

37. Bailey, T.L.; Johnson, J.; Grant, C.E.; Noble, W.S. The MEME Suite. Nucleic Acids Res. 2015. [CrossRef] [PubMed]

38. Mitchell, A.; Chang, H.-Y.; Daugherty, L.; Fraser, M.; Hunter, S.; Lopez, R.; McAnulla, C.; McMenamin, C.; Nuka, G.; Pesseat, S. The InterPro protein families database: The classification resource after 15 years. Nucleic Acids Res. 2014. [CrossRef] [PubMed]

39. Dai, X.; Zhao, P.X. psRNATarget: A plant small RNA target analysis server. Nucleic Acids Res. 2011, 39, W155-W159. [CrossRef] [PubMed]

40. Song, A.; Lu, J.; Jiang, J.; Chen, S.; Guan, Z.; Fang, W.; Chen, F. Isolation and characterisation of Chrysanthemum crassum SOS1, encoding a putative plasma membrane $\mathrm{Na}^{+} / \mathrm{H}^{+}$antiporter. Plant Biol. 2012, 14, 706-713. [CrossRef] [PubMed]

41. Song, A.; An, J.; Guan, Z.; Jiang, J.; Chen, F.; Lou, W.; Fang, W.; Liu, Z.; Chen, S. The constitutive expression of a two transgene construct enhances the abiotic stress tolerance of chrysanthemum. Plant Physiol. Biochem. 2014, 80, 114-120. [CrossRef] [PubMed]

42. Song, A.; Zhu, X.; Chen, F.; Gao, H.; Jiang, J.; Chen, S. A chrysanthemum heat shock protein confers tolerance to abiotic stress. Int. J. Mol. Sci. 2014, 15, 5063-5078. [CrossRef] [PubMed]

43. Song, A.; Li, P.; Jiang, J.; Chen, S.; Li, H.; Zeng, J.; Shao, Y.; Zhu, L.; Zhang, Z.; Chen, F. Phylogenetic and transcription analysis of chrysanthemum WRKY transcription factors. Int. J. Mol. Sci. 2014, 15, 14442-14455. [CrossRef] [PubMed]

44. Rozen, S.; Skaletsky, H. Primer3 on the WWW for general users and for biologist programmers. Methods Mol. Biol. 2000, 132, 365-386. [PubMed]

45. Livak, K.J.; Schmittgen, T.D. Analysis of relative gene expression data using real-time quantitative PCR and the $2^{-\Delta \Delta C t}$ Method. Methods 2001, 25, 402-408. [CrossRef]

46. De Hoon, M.J.; Imoto, S.; Nolan, J.; Miyano, S. Open source clustering software. Bioinformatics 2004, 20, 1453-1454. [CrossRef] [PubMed]

47. Eisen, M.B.; Spellman, P.T.; Brown, P.O.; Botstein, D. Cluster analysis and display of genome-wide expression patterns. Proc. Natl. Acad. Sci. USA 1998, 95, 14863-14868. [CrossRef] [PubMed]

(C) 2016 by the authors; licensee MDPI, Basel, Switzerland. This article is an open access article distributed under the terms and conditions of the Creative Commons by Attribution (CC-BY) license (http:/ / creativecommons.org/licenses/by/4.0/). 